\title{
The masses and radii of HD 186753B and TYC7096-222-1B: the discovery of two M-dwarfs that eclipse A-type stars (Research Note)
}

\author{
S. J. Bentley ${ }^{1}$, B. Smalley ${ }^{1}$, P. F. L. Maxted ${ }^{1}$, C. Hellier ${ }^{1}$, D. M. Wilson ${ }^{1,2}$, D. R. Anderson ${ }^{1}$, A. Collier Cameron ${ }^{3}$,

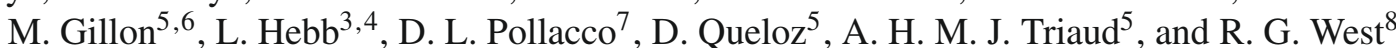 \\ 1 Astrophysics Group, Keele University, Keele, Staffordshire ST5 5BG, UK \\ e-mail: sjb@astro.keele.ac.uk \\ 2 Centre for Astrophysics \& Planetary Science, School of Physical Sciences, University of Kent, Canterbury, Kent CT2 7NH, UK \\ 3 School of Physics and Astronomy, University of St. Andrews, North Haugh, Fife KY16 9SS, UK \\ 4 Vanderbilt University, Department of Physics and Astronomy, Nashville, TN 37235, USA \\ 5 Observatoire de Genève, Université de Genève, 51 Chemin des Maillettes, 1290 Sauverny, Switzerland \\ ${ }^{6}$ Institut d'Astrophysique et de Géophysique, Université de Liège, Allée du 6 Août 17, Bat. B5C, Liège 1, Belgium \\ 7 Astrophysics Research Centre, School of Mathematics \& Physics, Queens University, University Road, Belfast BT7 1NN, UK \\ 8 Department of Physics and Astronomy, University of Leicester, Leicester LE1 7RH, UK
}

Received 20 August 2009 / Accepted 1 October 2009

\section{ABSTRACT}

\begin{abstract}
We present observations of two new single-lined eclipsing binaries, both consisting of an Am star and an M-dwarf, discovered by the Wide Angle Search for Planets transit photometry survey. Using WASP photometry and spectroscopic measurements we find that HD 186753B has an orbital period of $P=1.9194$ days, a mass of $M=0.24 \pm 0.02 M_{\odot}$ and radius of $R=0.31_{-0.06}^{+0.06} R_{\odot}$; and that TCY7096-222-1B has an orbital period of $P=8.9582$ days, a mass of between 0.29 and $0.54 M_{\odot}$ depending on eccentricity and radius of $R=0.263_{-0.07}^{+0.02} R_{\odot}$. We find that the Am stars have relatively low rotational velocities that closely match the orbital velocities of the M-dwarfs, suggesting that they have been "spun-down" by the M-dwarfs.
\end{abstract}

Key words. binaries: eclipsing - stars: early-type - stars: late-type - stars: individual: HD 186753 - stars: individual: TCY7096-222-1

\section{Introduction}

The radius of a star is one of its most fundamental properties, yet for sub-solar masses models have not been able to provide accurate radius predictions. Citing the discrepancies between model and empirical radius measurements, Chabrier et al. (2007) found that large surface spot coverage decreases the photospheric temperature. The star compensates by increasing its radius to conserve radiative pressure. This was confirmed by an empirical activity-radius study by Lopez-Morales (2007). In addition, Berger et al. (2006) found a correlation between an increase in metalicity and a larger-than-expected radius.

Because of their low intrinsic brightness, low-mass stars (LMS) are particularly difficult to study. LMS in eclipsing binary systems (EBLM), however, provide a direct way to obtain radius measurements and are therefore a valuable tool for testing models of stellar structure in the low-mass region. A by-product of wide-angle transit photometry planet-searching projects is the discovery of EBLMs (e.g. Fernandez et al. 2009).

The metallic-line Am stars are a class of peculiar A-type stars that are slow rotating, thought to have had their rotational velocity reduced by a near stellar companion. Spectroscopic orbits of many Am stars have been reported (e.g. Carquillat \& Prieur 2007; Renson \& Manfroid 2009) and LMS are thought to be responsible for reducing the rotational velocity of Am stars (Carquillat \& Prieur 2007). Here we report the discovery of two single lined A-M binaries, HD 186753 and TYC7096-222-1, the first EBLMs discovered from the Wide Angle Search for Planets (WASP) planet-hunting project. These are four likely eclipsing A-M systems that have previously been announced; three of these systems were announced in Dreizler et al. (2002) and one in Pont et al. (2005).

\section{Observations}

HD $186753(V=8.82)$ and TYC7096-222-1 $(V=10.34)$ were identified to be eclipsing systems by analysis of time series photometry observed between May 2006 and May 2008 (HD 186753) and May 2004 and March 2008 (TYC7096222-1) by the WASP-South observatory, totalling 11771 and 11879 photometric measurements, respectively. The WASPSouth passband has an effective wavelength of $581 \mathrm{~nm}$ with a passband width of $225 \mathrm{~nm}$, which is effectively a combination of the Johnson-Cousins $V$ and $I$-bands. Data were reduced with the WASP pipeline, de-trending and analysis tools described in Pollacco et al. (2006) and Collier Cameron et al. (2007). HD 186753 showed a recurrent eclipse signature with a depth of 0.015 mag and a period of 1.9194 days, whilst TYC7096-222-1 showed an eclipse depth of 0.024 magnitudes and a period of 8.9582 days, as shown in Fig. 1.

In October 2008 we obtained radial velocity measurements of HD 186753 and TYC7096-222-1 using the grating spectrometer on the 1.9-m telescope at the South African Astronomical Observatory (SAAO) at Sutherland, South Africa. HD 186753A 
Table 1. Radial velocity measurements of HD 186753 and TYC7096222-1.

\begin{tabular}{lll}
\hline \hline $\begin{array}{l}\text { HJD } \\
-2450000\end{array}$ & $\begin{array}{l}R V \\
\left(\mathrm{~km} \mathrm{~s}^{-1}\right)\end{array}$ & $\begin{array}{l}\sigma_{\mathrm{RV}} \\
\left(\mathrm{km} \mathrm{s}^{-1}\right)\end{array}$ \\
\hline HD 186753 & & \\
\hline $4617.83677^{\mathrm{H}}$ & 5.20 & 0.28 \\
$4657.17198^{\mathrm{C}}$ & -19.96 & 2.95 \\
4762.28479 & -40.05 & 3.33 \\
4763.26196 & 0.15 & 1.41 \\
4764.34678 & -44.69 & 3.72 \\
4765.29219 & 4.94 & 1.62 \\
4766.34139 & -44.74 & 3.72 \\
4767.36517 & 3.92 & 1.57 \\
\hline TYC7096-222-1 & & \\
\hline $4710.87886^{\mathrm{C}}$ & 21.86 & 0.08 \\
$4721.88521^{\mathrm{C}}$ & 10.98 & 0.10 \\
4763.56489 & 17.49 & 2.46 \\
4764.51536 & 17.95 & 2.49 \\
4765.52730 & 18.36 & 2.52 \\
4766.49429 & 11.88 & 2.05 \\
\hline
\end{tabular}

Superscript "H" denotes spectrum obtained by HARPS, "C" denotes CORALIE.

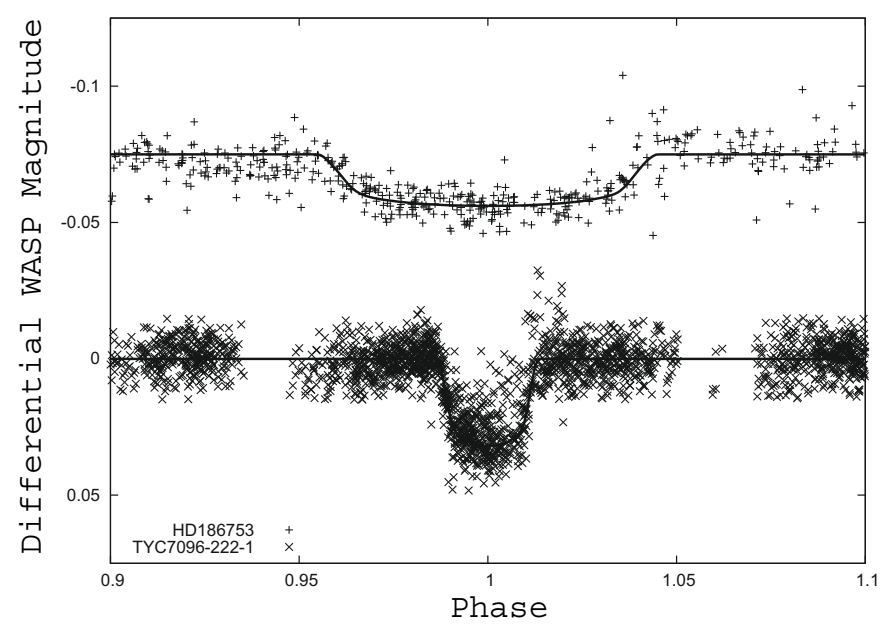

Fig. 1. The phase-folded WASP lightcurve of the eclipse of HD 186753A (offset by $0.075 \mathrm{mag}$ ) and TYC7096-222-1A overplotted with the best-fit models. The mean photometric uncertainty is 0.010 for HD 186753 and 0.018 for TYC7096-222-1.

has been identified by the SAO catalogue to be an A2 m spectral type, whilst we used the InfraRed Flux Method (Blackwell \& Shallis 1977) to estimate the spectral type of TYC7096222-1A as an F0. Because of this we used the spectral range 4666-4370 A that contains the Mg II $4481 \AA$ doublet, a spectral feature commonly used to obtain radial velocity measurements of early type stars. Two consecutive spectra were coadded and we used the Molly package to determine, by cross-correlation with a synthetic template, the radial velocity of the system. We obtained a single spectrum of HD 186753 from the CORALIE spectrograph on the 1.2-m Leonhard Euler telescope and a single spectrum from the HARPS spectrograph on the 3.6-m telescope at La Silla, Chile, on the nights of 31st August 2008 and 7th October 2008, respectively. We also obtained two spectra of TYC7096-222-1 from CORALIE on the nights of 31st August and September 11th 2008. We used the radial velocity standard star HD 8779 to calibrate the SAAO radial velocity data. The radial velocity data are shown in Fig. 2 and Table 1.

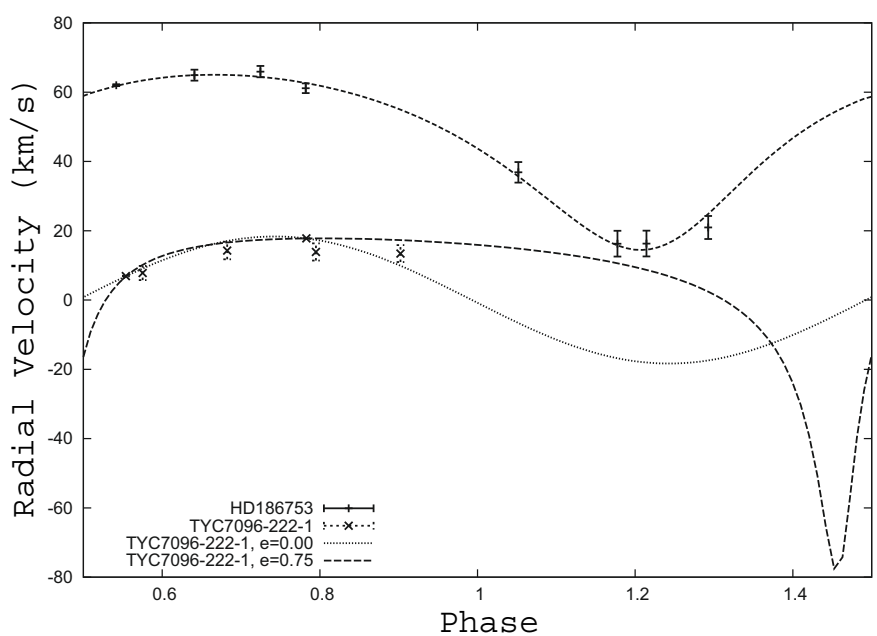

Fig. 2. The phase-folded radial velocity data of HD 186753 (offset by $50 \mathrm{~km} \mathrm{~s}^{-1}$ ) and TYC7096-222-1 overplotted with the best-fit models.

\section{System parameters}

For both targets we used the highest signal-to-noise (SNR) spectra taken by CORALIE $(\sim 50: 1)$ to estimate the stellar parameters of the primary. The low SNR has lead to relatively large errors on the final stellar parameters. Analysis of this spectrum was performed by the UCLSYN spectral synthesis package (Smith 1992; Smalley 2005) and ATLAS9 (Castelli et al. 1997) models without convective overshooting.

The $\mathrm{H}_{\alpha}$ and $\mathrm{H}_{\beta}$ lines were used to determine the effective temperature, $T_{\text {eff }}$. The equivalent widths of several clean and unblended lines were measured. Atomic line data was mainly taken from the Kurucz \& Bell (1995) compilation, but with updated van der Waals broadening coefficients for lines in Barklem et al. (2000) and $\log g f$ values from Gonzalez \& Laws (2000), Gonzalez et al. (2001) or Santos et al. (2004). The ionization balance between Fe I and Fe II was used to determine surface gravity, $\log g$. A value for microturbulence, $\xi_{\mathrm{t}}$, was determined from Fe I using Magain's (1984) method. Elemental abundances were determined from their measured equivalents. The quoted error estimates include that given by the uncertainties in $T_{\text {eff }}$, $\log g$ and $\xi_{\mathrm{t}}$, as well as the scatter due to measurement and atomic data uncertainties. The projected stellar rotation velocity $(v \sin i)$ was determined by fitting the profiles of several unblended Fe I lines.

For HD 186753A this fit yielded $T_{\text {eff }}=7800 \pm 200 \mathrm{~K}$, $\log g=4.0 \pm 0.3, v \sin i=65.0 \pm 5.0 \mathrm{~km} \mathrm{~s}^{-1}$ and $\xi_{\mathrm{t}}=$ $4.0 \pm 0.5 \mathrm{~km} \mathrm{~s}^{-1}$. HD 186753A is listed as having a spectral type A2mA5-F0 in Houck \& Smith-Moore (1988). Our analysis is consistent with this classification. Using the derived $T_{\text {eff }}$ from the spectrum and the stellar density, $\rho_{1}$, from the lightcurve analysis we used the isochrones of Girardi et al. (2000) to estimate a stellar mass of $M_{1}=1.79 \pm 0.05 M_{\odot}$ and WASP photometry to determine the radius as $R_{1}=2.53_{-0.30}^{+0.27} R_{\odot}$. For TYC7096$222-1 \mathrm{~A}$ we find $T_{\text {eff }}=7600 \pm 300 \stackrel{\mathrm{K}}{\mathrm{K}}, \log g=4.0 \pm 0.3$, $v \sin i=35.0 \pm 5.0 \mathrm{~km} \mathrm{~s}^{-1}$ and $\xi_{\mathrm{t}}=4.0 \pm 0.5 \mathrm{~km} \mathrm{~s}^{-1}$. Using the derived $T_{\text {eff }}$ and $\rho_{1}$ values with the Girardi et al. (2000) isochrones we find a mass of $M_{1}=1.74 \pm 0.05 M_{\odot}$ and radius of $R_{1}=1.66_{-0.08}^{+0.10} R_{\odot}$. The mass and radius of each primary is consistent within $1 \sigma$ of the empirical mass-radius measurements of A-type stars discussed in Torres et al. (2009). The results of the fit are shown in Table 2. 
Table 2. Parameters of HD 186753A and TYC7096-222-1A.

\begin{tabular}{lll}
\hline \hline Parameter & HD 186753A & TYC7096-222-1A \\
\hline Stellar Mass, $M_{1}\left(M_{\odot}\right)$ & $1.794 \pm 0.046$ & $1.735 \pm 0.054$ \\
Stellar Radius, $R_{1}\left(R_{\odot}\right)$ & $2.527_{-0.303}^{+0.270}$ & $1.662_{-0.081}^{+0.103}$ \\
Stellar Density, $\rho_{1}\left(\rho_{\odot}\right)$ & $0.367_{0.078}^{+0.119}$ & $0.375_{-0.051}^{+0.052}$ \\
$T_{\text {eff }}(\mathrm{K})$ & $7800 \pm 200$ & $7600 \pm 300$ \\
$\log g$ & $4.0 \pm 0.3$ & $4.0 \pm 0.3$ \\
$v \sin i\left(\mathrm{~km} \mathrm{~s}^{-1}\right)$ & $65.0 \pm 5.0$ & $35.0 \pm 5.0$ \\
$\left.\xi_{\mathrm{t}}(\mathrm{km} \mathrm{s})^{-1}\right)$ & $4.0 \pm 0.5$ & $4.0 \pm 0.5$ \\
{$[\mathrm{Fe} / \mathrm{H}]$} & $+0.12 \pm 0.12$ & $+0.08 \pm 0.13$ \\
{$[\mathrm{Ca} / \mathrm{H}]$} & $-0.41 \pm 0.14$ & $-0.63 \pm 0.09$ \\
{$[\mathrm{Sc} / \mathrm{H}]$} & $-0.65 \pm 0.12$ & $-1.47 \pm 0.05$ \\
{$[\mathrm{Y} / \mathrm{H}]$} & $+0.55 \pm 0.13$ & $+0.54 \pm 0.16$ \\
{$[\mathrm{Ba} / \mathrm{H}]$} & $+0.87 \pm 0.16$ & $+1.20 \pm 0.22$ \\
\hline
\end{tabular}

We used the systemic console (Meschiari et al. 2009) to fit our radial velocity data. The period was fixed to the period determined from WASP photometry, with phase $=1$ set at the centre of the eclipse. In our fitting procedure we assumed a Keplerian orbit of the M-dwarfs and fitted the stellar reflex velocity, $\mathrm{K}_{1}$, the centre-of-mass velocity, $\gamma$ and for HD 186753 we also fitted the eccentricity, $e$, and the longitude of periastron, $\omega$. The phase coverage of radial velocity data for TYC7096-222-1 is not sufficient for an accurate value of $e$. We therefore fixed $e$ to 0.75 , which is the highest eccentricity for an Am binary with a period between 4-12 days (Carquillat \& Prieur 2007) and left the remaining parameters free to fit to see what affect a high value of $e$ has on the secondary mass, $M_{2}$. The lightcurves were anylysed with the MCMC code described in Collier Cameron et al. (2007). The M-dwarfs were analysed as though they are dark objects as they both contribute to only $0.06 \%$ of light in the WASP passband.

We estimated the mass of the secondaries by using the inclination estimated from the MCMC analysis of the photometry and $K_{1}$ from the systemic console analysis of the radial velocity data. HD 186753B has a mass of $M_{2}=0.24 \pm 0.02 M_{\odot}$ and radius of $R_{2}=0.31_{-0.06}^{+0.06} R_{\odot}$. TYC7096-222-1B has a mass of $M_{2}=0.29 \pm 0.02 M_{\odot}$ and radius of $R_{2}=0.31_{-0.06}^{+0.06} R_{\odot}$, assuming zero eccentricity. Setting $e=0.75$ gives TYC7096-222-1B a mass of $M_{2}=0.54 \pm 0.06 M_{\odot}$. The results of the fit are shown in Table 3.

\section{Discussion}

The rotational velocity of A8 stars is expected to be $\sim 200 \mathrm{~km} \mathrm{~s}^{-1}$ (Gray 2005). The relatively low rotational velocities of HD 186753A and TYC7096-222-1A, $v \sin i=65.0 \pm 5.0$ and $35.0 \pm 5.0 \mathrm{~km} \mathrm{~s}^{-1}$, respectively, suggests that they have been "spun-down" by their M-dwarf companions. The stellar rotational angular velocity of HD $186753 \mathrm{~A}$ is $(3.67 \pm 0.65) \times$ $10^{-4} \mathrm{rad} \mathrm{s}^{-1}$ which is greater than the M-dwarf orbital angular velocity of $(3.79 \pm 0.01) \times 10^{-5} \mathrm{rad} \mathrm{s}^{-1}$. The rotational angular velocity of TYC7096-222-1A is $(3.16 \pm 0.47) \times 10^{-5} \mathrm{rad} \mathrm{s}^{-1}$ which is also greater than the M-dwarf orbital angular velocity of $(8.12 \pm 0.02) \times 10^{-6} \mathrm{rad} \mathrm{s}^{-1}$. The synchronisation timescales are 2.8 Myr for HD 186753 and 0.92 Gyr for TYC7096-222-1 (Zahn 1977), suggesting that each system is younger than the synchronisation time. Values of $\log g$ can be used as an age estimate, but our value is not reliable enough. Higher SNR spectra is required to determine the age of the systems.

The eccentricity of HD 186753B, $e=0.269 \pm 0.087$, is quite high for a short period binary and could also indicate

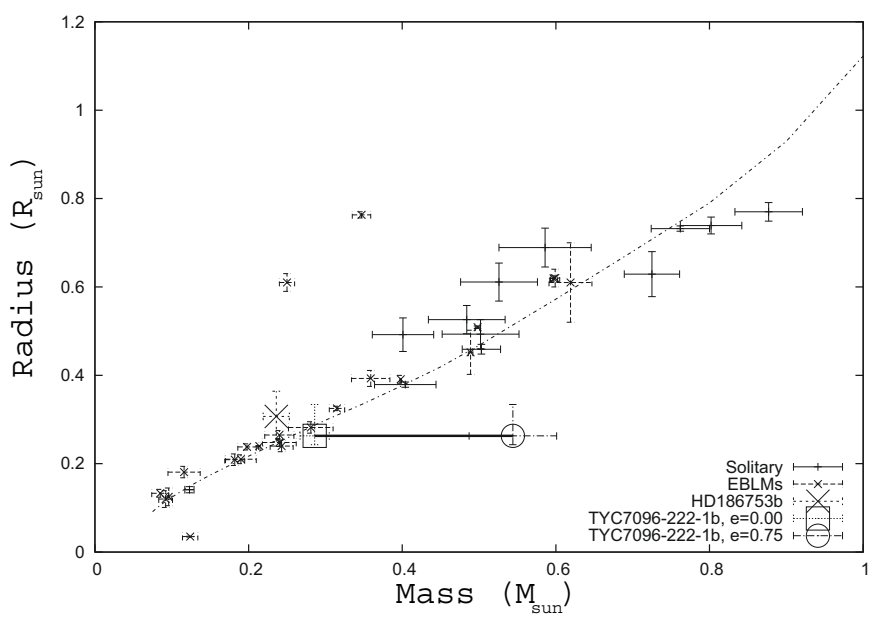

Fig. 3. Mass-radius relationship for solitary stars, EBLMs, HD 186753B and TCY7096-222-1 superimposed on 5 Gyr, $[\mathrm{M} / \mathrm{H}]=0$, $L_{\text {mix }}=1 H_{\mathrm{p}}$ Baraffe et al. (1998) isochrones. The thick black line indicates the range of masses for TYC7096-222-1B depending on the eccentricity. M-dwarfs with uncertainties in mass $>0.03 M_{\odot}$ have been omitted from the figure for illustrative clarity.

a young stellar age as the circulization time is 95 Myr (Zahn 1977). The circulization time for TYC7096-222-1B is 295 Gyr. If TYC7096-222-1B has a mass of $M_{2}=0.54 \pm 0.06 M_{\odot}$ (using the $e=0.75$ model) then we would expect a secondary eclipse of $0.003 \pm 1 \times 10^{-4} \mathrm{mag}$ deep. There appears to be no sign of this in the WASP photometry at any phase. TYC7096-222-1B therefore has a mass between $0.29-0.54 M_{\odot}$ depending on the eccentricity. The certainty of $e$ for both objects could be improved with greater radial velocity phase coverage. The value of $e$ for HD 186753B could be explained by a tertiary component either in the system or by a recent near-miss, although we find no evidence in our data for either scenario.

Both primaries show supersolar Fe abundances, an underabundance of $\mathrm{Ca}$ and $\mathrm{Sc}$ and overabundances of $\mathrm{Y}$ and $\mathrm{Ba}$. These abundances are typical of Am stars (e.g. Wolff 1983; Hundt 1972). Carquillat \& Prieur (2007) found that the mean companion mass to an Am spectral type is $0.8 \pm 0.5 M_{\odot}$ with a mean orbital period of 1.995 days. By these stellar properties HD 186753 and TYC7096-222-1 are fairly typical Am systems.

The radii of stars with masses below $0.3-0.35 M_{\odot}$ agree well with the Baraffe et al. (1998) isochrone models (Lopez-Morales 2007). The mass-radius relation TYC7096-222-1B agree within $1 \sigma$ of the Baraffe et al. (1998) isochrones, as shown in Fig. 3. Whilst the mass-radius relation of HD 186753B, however, is only just within $1 \sigma$ of the Baraffe et al. (1998) isochrones. M-dwarfs in binaries are found to be more active than solitary M-dwarfs (Lopez-Morales 2007). Increased activity causes a decrease in photospheric temperature, which then causes an increase in radius to conserve radiative pressure (Ribas 2006). $\mathrm{X}$-ray activity is an indicator of stellar activity, although no $\mathrm{X}$-ray data has been published on either object.

Being in a tight orbit, the photosphere of HD 186753B is strongly irradiated by HD 186753A. The radiation intensity received from HD 186753A at the photosphere of HD 186753B, and also therefore the radiative pressure, is $1.32 \pm 0.02$ times the radiation generated; the value for TYC7096-222-1 is lower at $0.72 \pm 0.04$. Higher precision radial velocity and photometry than the values given here are required to ascertain as to whether this extra source of heating, or increased levels of activity are affecting the mass-radius relation of the M-dwarfs. 
Table 3. Parameters of HD 186753B and TYC7096-222-1B and their orbits.

\begin{tabular}{|c|c|c|c|}
\hline Parameter & HD 186753B & TYC7096-222-1B $(e=0.00)$ & TYC7096-222-1B $(e=0.75)$ \\
\hline Eclipse epoch (HJD) & $3940.40144_{-0.00081}^{+0.00091}$ & $4373.01637_{-0.00087}^{+0.00066}$ & - \\
\hline Orbital period (days) & $1.9193851_{-0.0000393}^{+0.000081}$ & $8.9582591_{-0.0000314}^{+0.000037}$ & - \\
\hline Eclipse duration (days) & $0.1662_{-0.0031}^{+0.0038}$ & $0.2430_{-0.0042}^{+0.0039}$ & - \\
\hline Secondary/primary area ratio, $\left(R_{2} / R_{1}\right)^{2}$ & $0.0148_{-0.0003}^{+0.00031}$ & $0.0251_{-0.0005}^{+0.00022}$ & - \\
\hline Impact parameter, $b\left(R_{*}\right)$ & $0.264_{-0.154}^{+0.19003}$ & $0.250_{-0.142}^{+0.0 .0005}$ & - \\
\hline Stellar reflex vel., $\mathrm{K}_{1}\left(\mathrm{~km} \mathrm{~s}^{-1}\right)$ & $-27.449 \pm 1.751$ & $-20.341 \pm 2.974$ & $-58.432 \pm 6.122$ \\
\hline Centre-of-mass vel., $\gamma\left(\mathrm{km} \mathrm{s}^{-1}\right)$ & $-14.641 \pm 2.980$ & $4.074 \pm 1.533$ & $8.776 \pm 0.748$ \\
\hline Orbital separation, $a(\mathrm{AU})$ & $0.0370_{-0.0013}^{+0.0012}$ & $0.0990_{-0.0034}^{+0.0031}$ & - \\
\hline Orbital inclination, $i$ (deg) & $87.09_{-1.79}^{+1.00913}$ & $89.00_{-0.73}^{+0.0 .0034}$ & - \\
\hline Orbital eccentricity, $e$ & $0.269 \pm 0.087$ & $($ fixed $=0.00)$ & $($ fixed $=0.75)$ \\
\hline Longitude of periastron, $\omega(\mathrm{deg})$ & $166.7 \pm 5.9$ & (not fitted) & $167.2 \pm 61$ \\
\hline Stellar Mass, $M_{2}\left(M_{\odot}\right)$ & $0.236 \pm 0.017$ & $0.286 \pm 0.019$ & $0.544 \pm 0.057$ \\
\hline Stellar Radius, $R_{2}\left(R_{\odot}\right)$ & $0.307_{-0.057}^{+0.057}$ & $0.263_{-0.071}^{+0.020}$ & - \\
\hline Luminosity ratio, $L_{1} / L_{2}$ & $979.7^{-0.051}$ & $520.4^{-0.0 / 1}$ & 361.9 \\
\hline
\end{tabular}

The parameters of TYC7096-222-1B determined from the photometry are the same for both eccentricities.

Acknowledgements. S.J.B. acknowledges the support of an STFC postgraduate studentship. We are very grateful to the referee Jonathan Devor for his patience, assistance and helpful suggestions. The WASP consortium comprises the Universities of Keele, Leicester, St. Andrews, the Queens University Belfast, the Open University and the Isaac Newton Group. WASP-South is hosted by the South African Astronomical Observatory and we are grateful for their support and assistance. Funding forWASP comes from consortium universities and from the UKs Science and Technology Facilities Council. We also thank Tom Marsh for the use of his MoLLY spectra reduction programme.

\section{References}

Baraffe, I., Chabrier, G., Allard, F., et al. 1998, A\&A, 337, 403 Blackwell, D., \& Shallis, M. 1977, MNRAS, 180, 170

Barklem, P. S., Piskunov, N., \& O’Mara B. J. 2000, A\&AS 142, 467

Berger, D., Gies, D., McAlistar, A., et al. 2006, ApJ, 644, 475

Carquillat, J-.M., \& Prieur, J.-L. 2000, MNRAS, 380, 1064

Castelli, F., Gratton, R., \& Kurucz, R. 1997, A\&A, 324, 432

Chabrier, G., Gallardo, J., \& Baraffe, I. 2007, A\&A, 472, L17

Collier Cameron, A., Wilson, D., West, R., et al. 2007, MNRAS, 380, 1280

Demory, B.-O., Segransan, D., Forveille, T., et al. 2009, A\&A, in press

Dreizler, S., Rauch, T., Hauschildt, P., et al. 2002, A\&A, 391, L17

Fernandez, J., Latham, D., Torres, G., et al. 2009, ApJ, in press
Girardi, L., Bressan, A., Bertelli, G., et al. 2000, A\&AS, 141, 371

Gonzalez, G., \& Laws, C. 2000, AJ, 119, 390

Gonzalez, G., Laws, C., Tyagi, S., \& Reddy, B. E. 2001, AJ, 121, 432

Gray, D. 2005, The Observation and Analysis of Stellar Photospheres (Cambridge University Press)

Houk, N., \& Smith-Moore, M. 1988, Michigan Catalogue of Two-dimensional Spectral Types for the HD Stars, 4

Hundt, E. 1972, A\&A, 21, 413

Kurucz, R. L., \& Bell, B. 1995, Kurucz CD-ROM 23: Atomic Line List, SAO, Cambridge, USA

Lopez-Morales, M. 2007, ApJ, 660, 732

Magain, P. 1984, A\&A, 134, 189

Meschiari, S., Wolf, A., Rivera, E., et al. 2009, PASP, 121, 1016

Pollacco, D., Skillen, I., Collier Cameron, A., et al. 2006, PASP, 118, 1407

Pont, F., Bouchy, F., Melo, C., et al. 2005, A\&A, 438, 1123

Press, W., Teukolsky, S., Vetterling, W., et al. 1992, Numerical Recipes in C, The Art of Scientific Computing (Cambridge University Press)

Renson, P., \& Manfroid, J. 2009, A\&A, 498, 961

Ribas, I. 2006, Ap\&SS, 304, 89R

Santos, N. C., Israelian, G., \& Mayor, M. 2004, A\&A, 415, 1153

Smalley, B., Smith, K., \& Dworetsky, M. M. 2001, UCLSYN Userguide

Smith, K. C. 1992, Ph.D. Thesis, University of London

Torres, G., Andersen, J., \& Gimenez, A. 2009, A\&ARv, in press

Wolff, S. 1983, The A-Stars: Problems and Perspectives, NASA SP-463

Zahn, J.-P. 1977, A\&A, 57, 383 